\title{
Real-Time Range Imaging in Health Care: A Survey
}

\author{
Sebastian Bauer ${ }^{1}$, Alexander Seitel ${ }^{2,3}$, Hannes Hofmann ${ }^{4}$, Tobias Blum ${ }^{5}$, Jakob \\ Wasza $^{1}$, Michael Balda ${ }^{4}$, Hans-Peter Meinzer ${ }^{3}$, Nassir Navab ${ }^{5}$, Joachim Hornegger ${ }^{1}$, \\ and Lena Maier-Hein ${ }^{2,3}$ \\ 1 Pattern Recognition Lab, Friedrich-Alexander-Universität Erlangen-Nürnberg, \\ Erlangen, Germany, \\ sebastian.bauer@cs.fau.de \\ 2 Junior group: Computer-assisted Interventions, \\ 3 Division of Medical and Biological Informatics, German Cancer Research Center \\ (DKFZ), Heidelberg, Germany, \\ 1.maier-hein@dkfz-heidelberg. de \\ 4 Metrilus GmbH, Erlangen, Germany, \\ michael.balda@metrilus.de \\ 5 Computer Aided Medical Procedures \& Augmented Reality (CAMP), Technische \\ Universität München, Munich, Germany, \\ blum@in.tum.de
}

\begin{abstract}
The recent availability of dynamic, dense, and low-cost range imaging has gained widespread interest in health care. It opens up new opportunities and has an increasing impact on both research and commercial activities. This chapter presents a state-of-the-art survey on the integration of modern range imaging sensors into medical applications. The scope is to identify promising applications and methods, and to provide an overview of recent developments in this rapidly evolving domain. The survey covers a broad range of topics, including guidance in computer-assisted interventions, operation room monitoring and workflow analysis, touch-less interaction and on-patient visualization, as well as prevention and support in elderly care and rehabilitation. We put emphasis on dynamic and interactive tasks where real-time and dense 3-D imaging forms the key aspect. While considering different range imaging modalities that fulfill these requirements, we particularly investigate the impact of Time-of-Flight imaging in this domain. Eventually, we discuss practical demands and limitations, and open research issues and challenges that are of fundamental importance for the progression of the field.
\end{abstract}

\section{Introduction}

Computer assistance became increasingly important in health care over the last decades. Applications include computer-aided diagnosis, therapy support, virtual and augmented reality for intervention support and training, as well as systems to assist handicapped and elderly people. One of the key tasks for efficient computer-assistance in health care is a robust localization and tracking of the objects (operation situs, instruments) and persons (patient, physician, clinical staff) involved in the specific medical procedure. 
So far, this is typically performed using either (1) optical or electromagnetic tracking technologies that require markers to be attached to the target, or (2) by means of intra-operative radiographic imaging that implies a substantial radiation exposure to the patient and/or the physician. Marker-based approaches often complicate the clinical workflow and are thus not widely accepted in clinical routine.

In recent years, range imaging (RI) based techniques for marker-less, radiationfree localization have experienced a remarkable development with the availability of dynamic, dense and low-cost RI devices such as Time-of-Flight (ToF) cameras and Microsoft Kinect. Indeed, these modalities have been applied for numerous applications in the clinical environment, beyond marker-less localization. The scope of this state-ofthe-art survey is to give a comprehensive overview of the use of range imaging devices in the context of health care, with a focus on dynamic tasks that require real-time and non-scanning 3-D perception. To our knowledge, it is the first review to address the fast growing number of research activities in this area.

The remainder of this chapter is organized as follows. The main part of the survey divides into four fields of application: guidance in computer-assisted interventions (Sect. 2), monitoring for operation room safety and workflow analysis (Sect. 3), touchless interaction and on-patient visualization (Sect. 4), and diagnosis, prevention and support in screening, elderly care, rehabilitation and assistance for handicapped people (Sect. 5). In addition, we outline opportunities and limitations of different range imaging modalities, practical issues and dedicated software frameworks with a focus on the specific demands in medical applications (Sect.66. Eventually, we conclude with a discussion (Sect. 7) where we summarize the most substantial challenges that must be tackled to increase the range of potential applications in the particular field of health care, and identify future research directions.

\section{Guidance in Computer-assisted Interventions}

Guidance in computer-assisted interventions (CAI) is typically provided by establishing the spatial relationship between anatomical structures (acquired with some imaging modality prior to the intervention) and the medical instruments used during the intervention. This requires a registration of pre-operative patient-specific models to intra-operatively acquired data. One of the main challenges in this context is the fast, accurate, and robust acquisition of the patient anatomy during the intervention. Many CAI applications rely on modalities with limited imaging quality, such as ultrasound (US), or expensive and impracticable acquisition procedures, such as magnetic resonance imaging (MRI), or utilize custom designed markers that can be localized with optical or magnetic tracking systems. In contrast, real-time RI holds a simple, marker-less and non-ionizing alternative in interventional imaging. CAIs based on range imaging typically follow a generic workflow:

Treatment Planning: Prior to intervention, the patient's anatomy is acquired using standard medical imaging modalities such as computed tomography (CT) or MRI. Commonly, a treatment plan is derived from this data to be applied during intervention. Depending on the application, a simultaneous capture of range imaging data may be required during this planning stage.

Interventional Imaging: During the intervention, real-time RI allows for a continuous, marker-less and non-radiographic monitoring of the external body surface or the operation situs. 
Surface Registration: To transfer the treatment plan to the patient, the acquired RI surface is typically registered to a reference shape being extracted from the planning data before the intervention. This may involve the determination of the patient's orientation and pose, as well as non-rigid deformations induced by respiration, cardiac motion or interventional tissue manipulation. As this chapter is not intended to review surface registration techniques, we refer to dedicated surveys for more information $1,2,3,4,5$.

Guidance: The application of the treatment plan to the patient is accomplished by some sort of guidance that supports the physician during the intervention, e.g. by means of augmented reality (AR) visualization.

Below, we summarize applications and methods that have been proposed in the context of RI-based CAI for diagnostic and interventional imaging (Sect.2.1), radiation therapy (Sect. 2.2), tomographic reconstruction (Sect. 2.3), open and percutaneous interventions (Sect. 2.4), and 3-D endoscopy for minimally-invasive procedures (Sect. 2.5).

\subsection{Patient Setup in Diagnostic and Interventional Imaging}

In the past decade, substantial progress has been made in improving the image acquisition process in CT and MRI. However, optimizing the pre-imaging workflow has been considered only lately. In clinical practice, patient setup and scanner initialization including patient positioning, table adjustment, and the input of patient-specific parameters into the scanner software are performed manually, being both tedious and time consuming 6, 7. The automation of these steps would reduce both the examination time and the workload for clinical staff, thereby relieving the health care system. In CT imaging, the initial patient setup accounts for a substantial share of the entire procedure. To speedup the pre-imaging CT workflow, Schaller et al. proposed a marker-less system based on ToF imaging that identifies the coarse location of anatomical regions for prone and supine patient postures at interactive framerates 6 . The alignment of the pre-defined anatomical target with the scanner isocenter can then be performed in an automatic manner by either positioning the treatment table w.r.t. a non-moving acquisition device (CT/MRI), or by transforming a moving acquisition device (e.g. C-arm $\mathrm{CT}$ ) to coincide with the target. Note that a calibration between the coordinate systems of the RI camera and the scanner is required for this approach (cf. Sect. 6). Grimm et al. investigated the use of RI in the pre-imaging protocol for MRI [7]. In today's clinical routine, first, the patient orientation (head/feet first), posture (prone, supine, lateral left, right) and additional biometric information (body height, weight) must be specified by hand. Second, the radiologist manually defines the region of interest on the patient's body, typically using laser cross-hairs. To automate these tasks, Grimm et al. proposed a ToF-based system to detect both patient orientation and posture. In addition, using a model-based optimization framework, the articulated body pose of the reclined patient is estimated (Fig. 1 1a). This allows for a computerized localization of the scanning target, automating the pre-imaging workflow. Natural limitations involve the presence of blankets or additional equipment occluding the external patient surface, such as optional body coils in MR. In these cases, the localization must be performed prior to equipment placement.

\subsection{Positioning and Motion Management in Radiation Therapy}

The automation of patient setup is of particular interest for repeat treatments such as in fractionated radiation therapy (RT), where the tumor is irradiated in a sequence of 


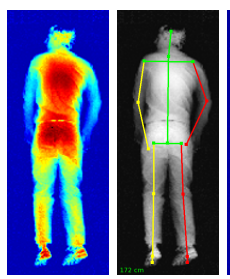

$(a)$

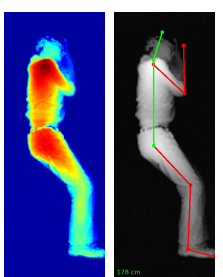

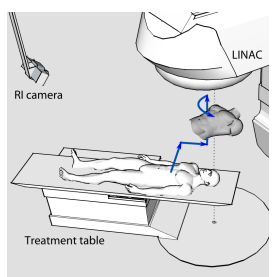

(b)

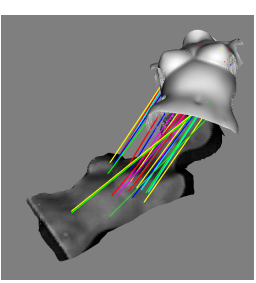

$(c)$

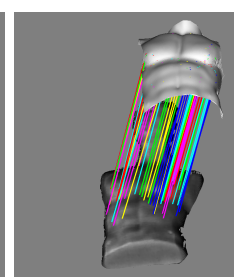

Fig. 1: (a) Estimating the body pose of a reclined patient on ToF data, for supine (left) and lateral left postures (right) [7. The skeleton of the estimated pose is overlaid, with the right and left extremities labeled in red and yellow, respectively. (b) Patient setup in RT, where the intra-fractional patient surface acquired with an RI camera is registered to a reference shape extracted from planning data (depicted in gray). The aligning transformation (in blue) is then applied to the treatment table. (c) Feature-based multi-modal surface registration between Microsoft Kinect (bottom) and CT data (top). [8]. The colored lines indicate the established point correspondences.

treatment sessions. Reproducible patient setup constitutes a key component for accurate dose delivery. Prior to each fraction, the target location known from tomographic planning data must be accurately aligned w.r.t. the isocenter of the treatment system (Fig. 1p). Conventionally, this alignment is performed in a two-step procedure, comprising (1) manual coarse patient setup using laser cross-hairs and skin markers, and (2) position verification and refinement using radiographic imaging. Over the past years, systems for non-radiographic patient setup and monitoring in RT have been proposed using different RI technologies, including active stereo vision [9], ToF imaging [10, structured light [8, 11, 12, and light sectioning [13, 14]. These systems estimate the rigid transformation that aligns the intra-fractionally acquired external body surface of the patient with a given reference shape extracted from tomographic planning data. This transformation can then be transferred to the treatment table control for automatic positioning. The focus of early solutions was on setup verification, restricting the automatic patient alignment to a fine-scale positioning and thus still requiring a manual setup initialization [9, 13. The first ToF-based systems for automatic patient setup in RT were proposed by Schaller et al. [15] and Placht et al. [10. However, these systems rely on rigid surface registration techniques and thus do not account for deformations induced by respiratory motion. To cope with this issue, Wasza et al. proposed a system for motion-compensated positioning based on patient-specific 4-D shape priors [1]. As the underlying iterative closest point (ICP) algorithm [16, 17] for these surface registration techniques is susceptible to local minima, the methods are restricted to resolving small initial misalignments. Targeting fully-automatic patient setup, Bauer et al. and Placht et al. proposed feature-based approaches that are capable to cope with gross initial misalignments 8,10 . Both rely on matching feature descriptors that encode the local surface topography. Point correspondences between the intra-fractional patient shape and a given reference then yield the aligning transformation (Fig. 1 More specifically, Placht et al. presented a mono-modal ToF-based solution where the intra-fractional patient shape is aligned to an RI reference shape acquired prior to the 


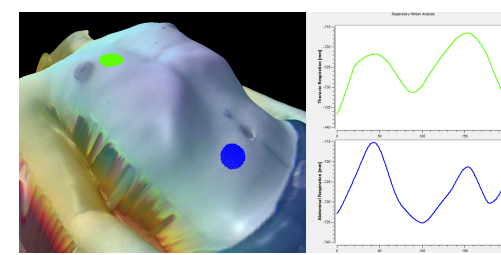

$(a)$

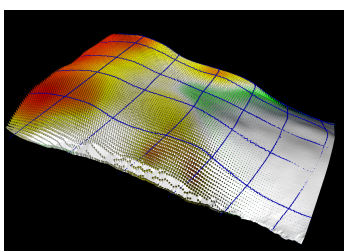

$(b)$

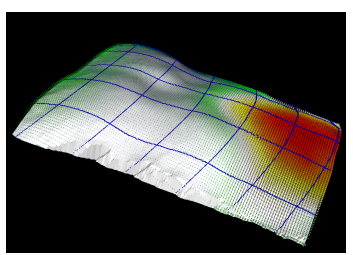

Fig. 2: (a) 1-D respiration surrogates extracted from Microsoft Kinect data, differentiating between thoracic (in green) and abdominal (in blue) motion. The target region (left) is set individually for each patient. (b) Dense surface deformation tracking [18] from sparse RI measurements (depicted in blue), using prior shape information from planning data (in gray). The magnitude of the local displacements is color-coded.

first fraction [10. Bauer et al. proposed a multi-modal surface registration scheme that enables a direct alignment of intra-fractional structured light data (Microsoft Kinect) to a reference shape extracted from pre-fractional tomographic planning data [8]. Both studies indicate the feasibility of the approach, yet being restricted to phantom experiments.

Real-time monitoring of the patient body holds great potential for the management of respiratory motion, being a rapidly evolving field in modern medicine. Motion management is of particular interest in image-guided RT for abdominal and thoracic targets where motion induces a substantial source of error. Accounting for potential targeting errors and to assure adequate dosimetric coverage of the tumor-bearing tissue, large safety margins are typically applied. However, this comes at the cost of irradiating surrounding radio-sensitive structures. To reduce tolerances between the planned and actually delivered dose distribution, a multitude of techniques for respiratory motion management have been developed over the past decades [19, 20]. Early strategies in RI-based motion tracking were restricted to low-dimensional respiration surrogates (cf. Fig. 2a). Schaller et al. presented a ToF-based system to acquire a low-dimensional respiratory signal 21. Lately, similar systems using Microsoft Kinect have been presented by Xia et al. and Alnowami et al. 22, 23. In contrast, recent RT motion tracking solutions target dense surface deformation tracking that better reflect the complexity of respiratory motion 24, 25. In combination with 4-D CT or MRI planning data, they can be used to establish patient-specific motion models [26] that correlate external body deformation with internal tumor motion. These models can then be applied for non-radiographic motion-compensated dose delivery. First approaches to reconstructing dense non-rigid torso deformations induced by respiratory motion were proposed only recently, (Fig. 2 p). Bauer et al. developed a joint variational formulation that simultaneously solves the intertwined tasks of denoising ToF data and its registration to a reference surface [27. Schaerer et al. studied the application of a non-rigid extension of the ICP algorithm with a commercially available stereo vision based RT solution 28. Further promising approaches for dense surface deformation tracking include sparse-to-dense shape registration based on a grid-type triangulation sensor [18, and photometry-driven surface registration [29]. Let us fur- 
ther remark that the analysis of dense displacement fields also allows for an automatic distinction between abdominal and thoracic respiration [30.

\subsection{Motion Compensation in Tomographic Reconstruction}

Beyond the discussed applications in RT, dense surface deformation tracking could also help reducing motion artifacts in tomographic reconstruction. Gianoli et al. proposed the use of marker-based surface tracking to extract a multi-dimensional respiration surrogate for reducing artifacts in retrospective 4-D CT image sorting [31. The experiments revealed that using multiple surrogates reduced uncertainties in breathing phase identification compared to conventional methods based on a mono-dimensional surrogate, cf. Sect. 2.2. In addition, RI-based body surface tracking is of particular interest for motion compensation in nuclear medical imaging such as positron emission tomography (PET) and single-photon emission computed tomography (SPECT) 32. Based on previous concepts for motion compensation in PET/SPECT using marker-based tracking 33, 34, 35, dense and real-time RI has been attracting interest in this field lately [36, 37. Open issues in this context such as the calibration and synchronization to the scanner are discussed in Sect. 6 .

\subsection{Guidance for Open and Percutaneous Interventions}

Guidance systems for open and percutaneous interventions require additional intraoperative imaging modalities to relate the present patient anatomy to the pre-operatively acquired planning data. Current systems use either radiographic imaging modalities or rely on tracking techniques that require additional markers to be attached to the instrument and the patient. To date, many of those systems have not become widely accepted in clinical routine because their benefit to the patient could not exceed the problems arising from the additional hardware complexity, radiation exposure and higher costs. Real-time RI constitutes an alternative for marker-less intra-operative acquisition of the operation area. In recent years, a variety of applications emerged that utilize range imaging cameras for intra-operative guidance, navigation and AR. One of the first approaches to assist open surgeries with marker-less guidance was presented by Cash et al. 38, 39. They proposed a system for image-guided liver surgery based on a laser range scanner and presented a method for recovering soft-tissue deformations using incomplete surface data 40. Although this concept was presented using a laser scanner for surface acquisition, it can be seen as the starting point for following research on the application of RI technologies in open surgery.

Mersmann et al. 42 investigated the suitability of ToF cameras as intra-operative modality for surface acquisition by comparing ToF and CT surfaces of explanted human and porcine organs. Furthermore, they investigated the use of ToF cameras as a markerless inside-out tracking device for AR visualization during image-guided procedures as opposed to the marker-based variant presented in 43. Dos Santos 44] presented a surface matching approach that allows for non-rigid intra-operative registration of ToF data.

Wang et al 45] proposed a needle tracking algorithm that is able to track standard biopsy needles within the field of view of the Microsoft Kinect camera without needing to attach any additional markers. Due to their thinness, biopsy needles can hardly be reconstructed in the depth map. Instead, the idea is to use the Kinect device as a stereo camera with the infrared and the RGB sensor forming the stereo pair. Based on 


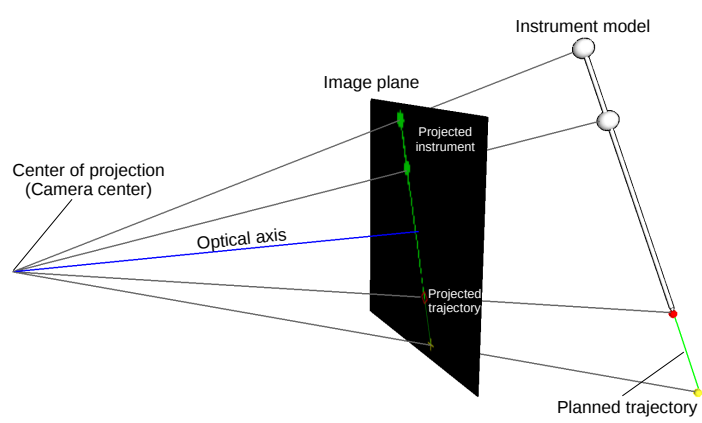

(a)

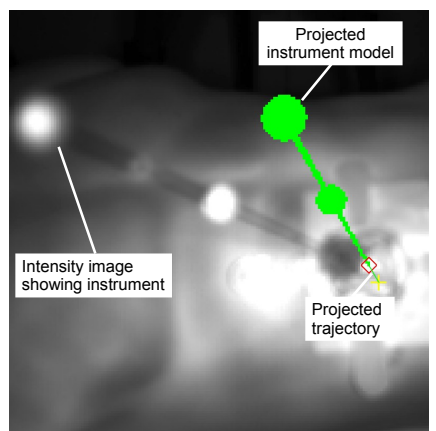

(b)

Fig. 3: Marker-less navigation concept for percutaneous needle insertions [41]. (a) Projection of the virtual needle model into the image plane of the RI camera. (b) AR view of the intensity image with projected final instrument position.

the needle being detected in both images separately, its 3-D pose can be estimated in four degrees of freedom. In a first evaluation the feasibility of the approach was shown. Navigated needle insertions have also been realized using range imaging techniques. Nicolau et al. [46] were one of the first groups to use a custom-made structured light camera system to assist percutaneous needle insertions. Having a video projector integrated into their structured light system, they were also able to project an AR view of internal organs directly onto the patient's surface. Seitel et al. 41] proposed another marker-less navigation approach for percutaneous needle insertions. Its main idea is to use an RI camera as a single modality for patient localization and instrument guidance. For guidance of the instrument, its virtual model is projected onto the image plane of the intensity/RGB image of the RI camera to provide guidance information during navigation, see Fig. 3 The accuracy of the presented approaches may not yet be sufficient for clinical use, however, along with a prospective increase in depth resolution, the integration of motion compensation methods and deformation models they hold great potential for future clinical applicability.

\subsection{3-D Endoscopy for Minimally Invasive Procedures}

While open surgery involves cutting the skin and dividing the underlying tissues to gain direct access to the surgical target, minimally-invasive surgery (MIS) is performed through small incisions in the skin in order to reduce surgical trauma. Laparoscopic surgery refers to MIS performed in the abdominal or pelvic cavities. As no direct view on the surgical target is possible, an endoscopic camera is used to provide a 2$\mathrm{D}$ view of the anatomical structures as well as the instruments applied. Due to the limited field of view, the difficult hand-eye coordination as well as the loss of depth perception and tactile feedback, laparoscopic interventions generally require a lot of skill and experience to be performed successfully. Hence, computer-assisted laparoscopy is subject of ongoing research. One of the main difficulties to be addressed is again the acquisition of the 3-D structure of the patient anatomy in an accurate, fast and robust manner during the procedure (cf. Sect. 2.4). Optical techniques for laparoscopic 3-D surface reconstruction can roughly be divided into two categories [47. Passive methods, 
such as stereoscopy [48]), shape-from-shading [49], shape-from-motion (SfM) [50], and simultaneous localization and mapping (SLAM) 51] need only endoscopic RGB images as input. Active methods, such as structured light [52, 53] and ToF require controlled light to be projected into the environment. For a comprehensive review of these different techniques in the context of MIS we refer to Maier-Hein et al. 54. In this chapter, we review recent advances related to ToF endoscopy.

While all the passive and active methods enumerated above have already been successfully applied in various fields, anatomic reconstruction for MIS poses several specific challenges: Firstly, the methods must be able to cope with a dynamic environment. Furthermore, human tissue often tends to be of homogeneous texture, making automatic feature detection and matching, required by most passive methods, difficult. Finally, miniaturization is necessary in order to build small devices that fit into the ports used in laparoscopic interventions. Behind this background, ToF imaging is a very interesting alternative for 3-D surface reconstruction in laparoscopic surgery, because it is real-time compatible, does not rely on salient features and does not require a baseline.

The first ToF-based endoscope was proposed by Penne et al. [55. The authors combined a commercial ToF camera featuring a lateral resolution of $64 \times 48$ px with a rigid standard endoscope optics. The standard illumination units of the ToF camera were replaced by a fiber-coupled high-power laser diode connected to the illumination fiber bundle of the endoscope. In a subsequent study, a higher resolution ToF camera featuring a lateral resolution of $204 \times 204$ px was used in a similar setup [56]. Recently, the company Richard Wolf GmbH (Knittlingen, Germany) introduced their first prototypical ToF endoscope. It features both a white light source as well as a ToF illumination unit and simultaneously generates range images $(64 \times 48 \mathrm{px})$, corresponding gray-scale amplitude images and standard definition RGB images $(640 \times 480 \mathrm{px})$ at a framerate of $\sim 30 \mathrm{~Hz}$. One application being addressed with these initial prototypes was laparoscopic instrument localization [57. Furthermore, first approaches to the fusion of endoscopic ToF and SfM [58] as well as SLAM [59] have been proposed.

In the context of endoscopy, the major advantages of ToF compared to other reconstruction techniques are the registered depth and intensity data at high framerates and the compact design without scanning component or baseline. However, the reconstruction accuracy of the first prototypical ToF endoscopes is not yet sufficient for clinical application, cf. Sect. 6.1 Still, due to the continuous technological advances related to ToF as well as the growing number of applications in various areas, ToF measurement precision and accuracy can be expected to increase further, thus making ToF endoscopy a new technique with high potential for computer-assisted endoscopy. Applications besides intra-operative registration for AR guidance are instrument tracking, collision avoidance in robotic-assisted MIS, and quantitative metric measurements.

\section{Monitoring for OR Safety and Workflow Analysis}

Monitoring the working area of operating rooms (OR) or intensive care units using a multi-camera setup of conventional cameras or RI cameras has attracted increasing attention lately 60, 61. The reason for this interest is twofold. First, it can help improve both medical staff and patient safety by monitoring human-robot interaction (Sect. 3.1). Second, it holds great potential to analyze and optimize the efficiency of clinical workflows (Sect. 3.2 . 


\subsection{Room Monitoring for Safety in Robot-assisted Interventions}

Collision avoidance in interventional environments is an emerging topic with the increased use of robotics in the OR. Real-time range imaging holds potential to ensure safe workspace sharing in this context. Let us point out the requirements for an RIbased collision avoidance system. First, it needs to have a low latency to cope with the dynamics of the scene. The particular demands in terms of latency and framerate can be derived from the given maximum velocity of the moving components in the scene. Second, as the scene of an OR during intervention is usually rather complex, occlusion will occur. Multiple cameras can be used to resolve the occlusion problem and provide additional redundancy in the data. However, note that RI technologies such as ToF or structured light suffer from signal interference that has to be coped with (cf. Sect.66). Another open topic is the optimal placement of cameras in a multi-camera setup to ensure sufficient coverage of the monitored workspace. On the other hand, one wants to use as few cameras as possible, because of interference, the amount of data to be processed, and the cost of the system. The overall system accuracy is limited by the individual sensor accuracy and the accuracy of the calibration of the sensors to each other. Since the moving components usually cannot be stopped instantaneously, a safety margin of the collision area in the centimeter range is mandatory. Consequently, the accuracy demands in human-robot collision avoidance scenarios are in that range as well.

The aim of the EU projects SAFROS 62 and ACTIVE 63 is to address the described problems. For instance, Mönnich et al. and Nicolai et al. proposed an OR supervision system based on multiple RI cameras [64, 65]. In particular, they used seven ToF cameras in order to monitor the scene from different perspectives. Based on extrinsic camera calibration, the system enables a volumetric reconstruction of the OR workspace.

\subsection{Monitoring, Analysis, and Modeling of Workflows}

Another research direction where RI cameras are of great interest is the modeling, recognition, analysis and interpretation of workflows and activities during surgery. A computer system that is able to understand activities inside the OR has several potential applications such as context-aware guidance and provision of user interfaces, relevance based visualization, monitoring of the operation for unexpected events, automatic documentation, or prediction of the remaining duration of a surgery. Most work in this area is based on the concept of recording several medical procedures and generating a statistical model of the workflow. Later, during a running medical procedure, intra-operative data from RI cameras is compared to this statistical model 66.

One example of using range images for workflow recognition has been shown by Padoy et al., using a real-time 3-D reconstruction of a simulated OR that was obtained from a multi-camera system 67. Based on the 3-D reconstruction, the motion flow of staff and objects inside the OR was computed and a statistical model was generated. This model allows detection of surgical phases during a running surgery. Lea et al. used a single RI camera to detect medical staff in a pediatric intensive care unit 68. They extracted features such as position, orientation and interaction between persons from the range images. Based on these features they recognized different actions during the intervention. There are also related applications that do not require a temporal workflow model. For instance, Ladikos et al. 69 used a real-time 3-D reconstruction of an OR to analyze radiation exposure during interventions. They recognize the position 
of OR staff with respect to an X-ray device and can model the radiation exposure over the course of an intervention. Compared to standard radiation counters the advantage of such a system is that the radiation exposure for each part of the body is simulated. For inexperienced OR staff it is also very interesting that after the intervention it can be visualized when and where the radiation exposure occurred to increase the awareness of the dangers of radiation.

\section{Touch-less Interaction and Visualization}

Real-time range imaging also holds potential for touch-less interaction in sterile environments (Sect. 4.1) and for on-patient visualization of medical data (Sect.4.2). In this section, we summarize the developments in these two emerging fields of application.

\subsection{Touch-less Interaction in Sterile Environments}

The recent advent of touch-less real-time user-machine interaction that came along with the introduction of low-cost RI sensors has also evoked interest in the medical domain. In particular, gesture control holds potential in areas such as interventional radiology, neurosurgery or navigated surgery where volumetric scans such as CT or MRI are commonly used for intra-operative guidance. There are two main arguments for using touch-less interaction in medical interventions. First, the surgeon has to remain sterile. This limits the usability of mouse and keyboard. Second, operating rooms are typically packed. Therefore, workstations allowing access to medical images usually require the surgeon to move away from the patient. Today, the surgeon commonly delegates the interaction with computers to nurses. However, this often leads to misunderstandings and delays, in particular for complex tasks. In general, there are different alternative solutions to range imaging for touch-less interaction such as data gloves, accelerometers, optical or magnetic tracking systems and hand recognition in color cameras. However, these solutions either require hardware or markers to be worn by the user, or are less robust. In the last decade, several systems for touch-less interaction using stereo cameras [70, ToF imaging [71] and recently Microsoft Kinect [72, 73, 74, 75, 176, 77] have been proposed. A commercially available system using infrared stereo cameras to access patient records in the OR is offered by Karl Storz GmbH, Tuttlingen, Germany [78]. For a more general overview on RI-based gesture recognition we refer to Chap. Gesture Interfaces with Depth Sensors.

As gesture-based interaction is not an established input method such as keyboards or touch-screens, finding appropriate interaction metaphors is still a topic of ongoing research. Existing prototypes use different concepts such as mapping the hand position to the position of the mouse pointer and using clicking gestures 70, 72. Other systems analyze gestures of the fingers [71, 73], the hands [73, 75, 74, 76, 79] or analyze the hand position w.r.t. the body [76. Most existing research prototypes allow navigation through either 2-D or 3-D image data. For 2-D images, the selection of slices, zoom and changing brightness and contrast are common operations that have been implemented. For 3-D image viewing, rotation and translation are common operations. Another functionality that has been implemented is the measurement of the size of a structure [71. Additional functions that have been tested in some prototypes are control of OR parameters such as turning on or off the light 70, 79. One common problem of gesture-based user interfaces is to differentiate between intended and unintended gestures. Different methods to address this issue have been proposed, such as 


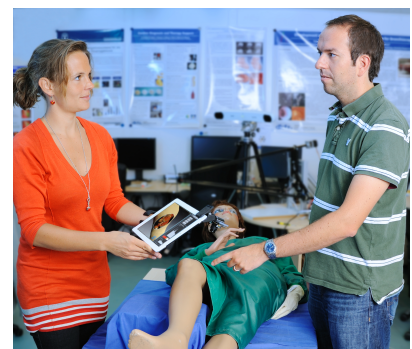

(a)

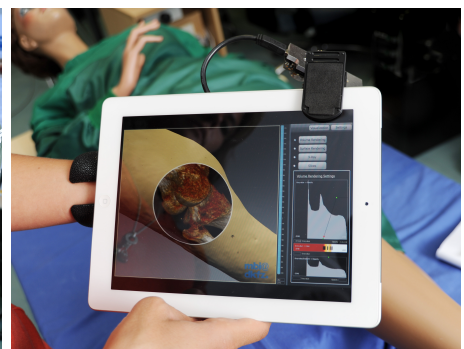

(b)

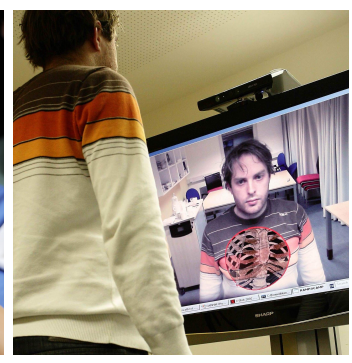

(c)

Fig. 4: Concept of on-patient visualization. (a,b) The pose of the display and thus the viewing direction of the user is continuously computed based on surface data captured with a range imaging device [83, (C) DKFZ, Tobias Schwerdt. (c) AR overlay of anatomical information in a magic mirror for education of anatomy [84].

using a specific interaction zone [70, 73, or distance 72, using certain gestures 71, using voice recognition to activate gesture recognition [76, 79] or analyzing the pose of the user with respect to the display 74 .

To evaluate the use of touch-less interactions for medical applications several studies have been conducted, involving participants without medical background [71, 75, 77, medical doctors in a simulated setup [76, 77] and medical doctors in real surgeries 80 . All studies reported results in favor of touch-less interaction. Most systems are still prototypes and research on using gesture-based interaction in real medical interventions has rarely been addressed. Nevertheless, gesture-based interaction holds great potential as there is an increasing need for user interfaces to operate computer-based systems in the OR and gesture-based user interfaces have substantial advantages over traditional interfaces.

\subsection{On-patient Visualization of Medical Data}

The visualization of anatomical data for the purpose of disease diagnosis, surgical planning, or orientation during interventional radiology and surgery is an integral part of modern health care. However, only few medical imaging modalities are capable of providing real-time images of the patient's anatomy. A common procedure therefore involves the acquisition of static 3-D image data, e.g. by means of CT or MRI scanners, and subsequent manipulation and visualization of the acquired data on a workstation. However, in such conventional techniques it is usually the task of the physician to mentally transfer the 3 -D virtual image to the patient, which requires considerable skill and experience. In addition, navigation in the three-dimensional data set may be rather cumbersome. To overcome these issues, methods for on-patient visualization during medical interventions via AR, using head-mounted displays 81] or intra-operative projector systems 82, for example, have been proposed. However, these AR systems are typically expensive, require the attachment of markers to the patient, or are difficult to integrate into the medical workflow due to bulky equipment. 
A novel alternative for intuitive and real-time on-patient visualization of anatomical data are RI devices that allow for capturing the patient anatomy without markers and in real-time. In particular, low-cost RI cameras hold potential for cost-sensitive applications such as medical education, training and rehabilitation. One promising application involves tracking the pose of a person in order to visualize subsurface anatomical detail via AR, as suggested by Maier-Hein et al. 83] and Blum et al. 84. Maier-Hein et al. 83. proposed mounting a ToF camera to a portable display or tablet for on-patient visualization of medical images, as shown in Fig. 4 a,b. The basic idea is to compute the pose of the mobile display relative to previously acquired 3-D tomographic data set by means of surface registration (cf. Sect. 2). Estimating the pose of the camera and thus the viewing direction of the physician allows for visualization of internal anatomical structures on the portable display as illustrated in Fig. $4 \mathrm{~b}$. In addition, navigation through medical imaging data becomes more intuitive because it is performed directly at the object of interest. As the quality of visualization depends on the accuracy of surface registration, the authors proposed an anisotropic ICP variant 85, 86] that accounts for the resolution and precision of RI devices in different directions. A trimmed version of the algorithm, which allows for aligning partially overlapping surfaces, has been also applied in this context 87.

Blum et al. 84 presented a system that involves tracking of a person in front of a large screen that serves as a kind of mirror (cf. Fig 4 .). Registration of the person's body surface, captured with an RI device, with a virtual patient model allows for AR visualization of subsurface anatomical detail in the mirror. The system can be applied for education purposes as it provides an intuitive visualization of anatomical information. It can be further used to support patient-doctor communication, based on the visualization of patient-specific data. Note that instead of surface registration between the medical data and the RI-based shape of the user, full-body motion capture methods (cf. Chaps. Full-Body Human Motion Capture from Monocular Depth Images and A Survey on Human Motion Analysis from Depth Data) may be applied.

\section{Diagnosis, Prevention and Support}

In this section, we summarize the developments in diverse fields of applications. More specifically, we review the use of real-time range imaging in elderly care (Sect. 5.1), early diagnosis and screening (Sect. 5.2), rehabilitation (Sect. 5.3), and support for handicapped people (Sect. 5.4.

\subsection{Activity Assessment in Elderly Care}

In-home activity assessment in elderly care is a rapidly evolving field. The need for care facilities and the associated costs for the health insurance system can be alleviated by low-cost systems that allow older adults to continue life in independent settings. These systems focus on monitoring the health status, sharing information about presence and daily activities, and providing on-line assistance and coaching. Low-cost range imaging holds great potential in this context. For instance, recognition of early indicators of functional decline such as deviations in gait using RI-based pose estimation 88 can help preventing accidents, automatic detection of abnormal events such as falls 89 can improve the respond time in emergency situations, and retrospective data analysis can help understand the mechanisms that led to an event. In elderly care, both static 
installations 90, 91, 92, 93, and mobile robotic platforms [94, 95, 96] that incorporate dense and real-time range imaging have been proposed. Most systems that rely on human pose analysis exploit the mass-market proven skeletal tracking that ships with Microsoft Kinect [97]. ToF-based pose estimation has been rarely considered [98, 99.

\subsection{Early Diagnosis and Screening}

The detection of abnormal behavior based on range imaging technologies also holds potential for early diagnosis and screening, for different groups of patients. Information about daily lifestyle and deviations from the normal can help in early diagnosis or progression analysis for cognitive impaired people such as Alzheimer's [100] or Parkinson's disease patients [88. Low-cost RI devices further open the possibility of large-scale screening of at-risk groups. For instance, in developmental disorders such as autism and schizophrenia, observing behavioral precursors in early childhood using 3-D perception for activity recognition [101, 102] can allow for early intervention and thus improve patient outcomes. In sleep monitoring, range imaging is gaining interest for non-contact measurement of sleep conditions or diagnosis of sleep apnea, for instance using ToF [103] or structured light [104] range imaging.

\subsection{Treatment Support in Rehabilitation}

RI sensors have also attracted interest in the field of physical therapy. The basic idea of using serious games in rehabilitation is to increase motivation and engagement of the patient, thus improving exercise performance, perseverance and rehabilitation outcomes 105. RI-based games are of particular interest, as the embedded sensors simultaneously allow for a quantitative assessment of performance. Hence, the rehabilitation progress can be tracked and analyzed to modify the therapy, if necessary. Furthermore, the workload of professional therapists is reduced. Low-cost RI systems have lately been considered for tele-rehabilitation techniques [106, 107] that are beneficial for translating skills learned in therapy to everyday life. Tele-rehabilitation systems allow the therapist to monitor the patient during exercising at home, track their activity and progress, and provide feedback. Recently, RI-based rehabilitation systems for physically disabled patients [108, 109, 110, 111, chronic pain patients [112] and patients after neurological injuries [113, 114] have been proposed. The vast majority of approaches in the field of serious games builds on Microsoft Kinect, being inherently embedded in an off-the-shelf gaming console.

\subsection{Aids for Handicapped People}

Recently, first approaches towards the use of assistive technologies to support handicapped people were proposed 115 . The integration of an RI device into an augmented blindman's cane or head-mounted systems could aid visually impaired people in navigation by identifying and describing surroundings beyond the limited sensing range of a physical cane [16, 117, 118, 119. For instance, Gassert et al. [117] described the augmentation of a white cane with a ToF sensor. Low-cost range imaging has been also proposed for autonomous transportation vehicles that follow handicapped people using 3-D perception [120]. 


\section{Practical Issues}

Medical applications impose several practical demands on RI cameras, including realtime capability and a high degree of absolute accuracy, reliability, and robustness. However, RI data are typically prone to inaccuracies due to technological limitations. In this section, we identify issues that are specific for applications in health care (Sect. 6.1. Dedicated software frameworks for RI processing that explicitly address medical applications are briefly outlined in Sect. 6.2

\subsection{Issues and Limitations}

First and foremost, the enhancement of RI measurement data in terms of denoising and correction of modality-specific systematic errors - such as intensity- or temperaturerelated distance errors, motion artifacts, and outliers at depth discontinuities in ToF imaging - assumes a critical role in the processing pipeline. In theory, some of these systematic errors could be compensated for by a calibration procedure performed once before clinical use. For ToF-based systems, a practical approach would further assume a constant temperature after a warm-up period and a fixed integration time that must be chosen in an application-specific manner. However, even though progress has been made in understanding the underlying technological issues, a robust and holistic calibration and correction of systematic errors is still an open field of research. This particularly applies for the young discipline of ToF imaging, for a comprehensive treatment we refer to Chap. Technical Foundation and Calibration Methods for Time-of-Flight Cameras. Here, the focus is on practical aspects that are specific for medical applications. Thus we conclude that the quality of RI data is a limiting factor concerning the spectrum of medical applications that can be potentially addressed. Indeed, the achievable degree of accuracy of today's RI cameras hinders a more widespread usage.

The acquisition of RI data in a medical context poses several challenges. The systems typically face a dynamic and often unpredictable environment, and occlusions that result from clinical staff or interventional devices such as linear accelerators or C-arm CT systems that may temporally obstruct the field of view of the RI camera (cf. Sect. 2). Yet, even in a static scenario with known acquisition geometry, small reconstruction frustums, shallow acquisition angles or a large working distance may deteriorate the quality of RI data or invoke self-occlusions. Applications where the patient is partially covered by a blanket or equipment are even more challenging or impossible to address with RI.

For many clinical scenarios, using a multi-camera acquisition setup can help to improve the scene coverage in both dynamic and static environments (cf. Sect. 3). However, this typically entails substantial efforts w.r.t. robust and recurrent intrinsic and extrinsic calibration, potentially introducing an additional error source. Furthermore, available RI technologies such as ToF or structured light are known to suffer from issues due to signal interference. For ToF imaging, several techniques to suppress interference have been investigated, such as modulation frequency or code division multiplexing (see 121 for an overview). For structured light, the usage of different light frequencies for the pattern can be used. Maimone et al. 122 have proposed a multiple Kinect system that involves vibrating the Kinects at distinct frequencies. If the cameras support a framerate that is substantially higher than the overall required framerate, temporal multiplexing with an external trigger may be used. In addition to mutual signal interference in a multi-camera setup, the influence of the infrared 
part of ambient or high-intensity surgical lighting on RI reconstruction robustness and accuracy should be investigated.

Besides acquisition constraints in a medical environment, surface and tissue properties of the observed object assume a decisive role. In particular for ToF-based open surgery (Sect. 2.4) and endoscopic procedures (Sect. 2.5), translucent and glossy organ surfaces impair RI measurements. For instance, specular highlights may cause invalid depth measurements due to sensor saturation. Furthermore, reflective or absorbing tissues and fluids such as blood 123 may lead to multi-path or sub-surface penetration, signal attenuation and scattering issues with ToF imaging [124, 125, 126] that might impair both absolute accuracy and signal-to-noise ratio. These are critical issues to be addressed in future work. Note that similar effects occur with applications that involve range imaging of the external body surface. In this context, different skin types may influence the range measurements.

Another important issue for the application of RI technologies in health care concerns a proper integration into clinical routine. The need for a warm-up period for ToF devices, frequent re-calibration procedures for multi-camera setups, or frequent re-calibration between the sensor and scanner coordinate systems might be problematic for certain clinical workflows. For patient setup and monitoring solutions, system calibration w.r.t. a treatment couch coordinate system has been shown to be manageable. For instance, the VisionRT system being a widely established range imaging system in RT relies on a calibration with a dedicated pattern [127. In contrast, for calibrating the RI coordinate system w.r.t. imaging modalities such as CT, MRI, US, PET or SPECT, the design of customized calibration patterns may be necessary. This also applies for the joint application of RI-based and conventional tracking solutions using optical or electromagnetic markers. In addition, let us stress that the accuracy of system calibration w.r.t. different modalities and coordinate systems highly depends on the accuracy and reliability of the RI measurement data itself.

For RI-based guidance in computer-assisted intervention, an open field of research beyond calibration concerns the relation of the measured external topography to the internal structures given from tomographic data $(\mathrm{CT} / \mathrm{MR})$. In clinical practice, the external shape acquired with an RI system during intervention typically does not coincide with the shape extracted from tomographic planning data due to non-rigid deformations that occur due to body twist and bend, respiration, cardiac motion, or tissue manipulation. Promising future directions involve the use of generic or patientspecific models that correlate external motion to internal motion based on dynamic 4-D CT/MR data 24, 25]. Only little research has investigated the use of biomechanical models propagating surface deformations to the internal structures known from a static tomographic scan.

Range imaging systems that are intended to be used in surgical and endoscopic interventions need to be compact. This implies several restrictions for the different range imaging principles, such as miniaturized illumination units for ToF sensors in general, sufficient light transmission for 3-D ToF endoscopy and a small baseline for structured light sensors. In the context of endoscopic applications in MIS, errors caused by background illumination can be neglected due to the controlled environment. On the other hand, working in a cavity of reflecting tissue poses challenges regarding multipath reflexions. Furthermore, it is a great challenge to transmit enough light to the tissue, which leads to a low signal-to-noise ratio in endoscopic ToF images and hence a decreased measurement accuracy in camera direction compared to standard ToF cameras. It is theoretically possible to increase measurement accuracy by operating 
the ToF device with a higher modulation frequency 128. Due to the small working volume in laparoscopic interventions, the reduced ambiguity range would be acceptable.

In conclusion, we stress that the integration of range imaging systems into clinical routine is restricted due to the lack of certified standard hardware or the early prototype stage of dedicated devices such as in 3-D endoscopy. This hinders experimental studies involving patients and, thus, most research on surgical and endoscopic applications relies on synthetic phantom or animal studies.

\subsection{Software Frameworks}

Two open-source frameworks have addressed range imaging in medical applications so far: MITK-ToF 129 as an integration into the well-known Medical Imaging Interaction Toolkit (MITK) 130 and the Range Imaging Toolkit (RITK) 131. Both frameworks build upon the Insight Segmentation and Registration Toolkit (ITK) 132, which is considered as de-factor standard in open-source medical image processing. Whereas the focus of MITK-ToF is more on the medical integration and interaction with other modules, RITK has a strong focus on hardware accelerated multi-view range data streaming to satisfy real-time demands in clinical practice, e.g. using general purpose computing on graphics processing units (GPGPU). As a general RI framework, the Point Cloud Library [133] has become popular in the computer vision community.

\section{Discussion}

In this chapter we have given an overview of the application of range imaging in different fields of health care. While some are merely in a proof of concept state and still require basic research to be done, others are already close to being employed in commercial products. Most often, RI is used for localization and tracking of objects and persons in 3 -D workspace. Commercially available systems that solve these tasks utilize markerbased solutions or radiographic imaging. However, attaching markers is time-consuming and complicates the workflow. Radiation on the other hand is harmful to both the patient and medical staff. The main advantages of RI cameras are that they operate touch-less (sterile), marker-free (no setup required), fast (real-time), and dense (nonscanning).

Range imaging can help improve health care in many areas. Outside a clinical environment, even at home, body tracking and pose detection can support prevention, rehabilitation, and remote diagnosis. Location and pose information is also required for automatic patient positioning. When tracked over time, information about nonrigid surface deformations can be used to compensate for patient motion, e.g. in tomographic reconstruction, or radiation therapy. With additional instrument tracking, guidance is viable. Robust localization and 3-D surface information is also the basis for AR applications that hold potential in interventional navigation as well as humandoctor communication. Touch-less gesture recognition promises to solve the problem of sterile human-machine-interaction in the OR. The technology is mature, however, its widespread acceptance is hampered by the lack of a common, intuitive set of gestures. Finally, 3-D endoscopy and room supervision are areas where range imaging creates new types of data. Both hold great potential, but still require considerable research.

Future research on real-time RI in health care should cover three areas. First, there are several open research questions which have to be tackled. Since the underlying technologies are relatively new and still immature to some degree, available RI cameras have 
shortcomings, which have to be resolved (e.g. systematic errors, low spatial resolution). Further, the registration of RI measurements to data acquired with conventional medical imaging modalities is important. Other open questions regard the adequacy of the acquired data, e.g. whether external surface information is sufficient for tracking internal structures, or whether the achievable absolute accuracy is acceptable for a given application. Second, everyday issues of current applications and prototypes must be solved. This involves their integration into clinical scenarios and workflows, including e.g. multi-camera setups, calibration and synchronization to a scanner or treatment system, and real-time implementation of algorithms. Third, medical certification for $\mathrm{RI}$ devices and related clinical applications is another fundamental requirement for the progression of the field. Once these issues have been solved and range imaging technology has been established in daily health care routine, it will lead to new, more efficient and safe workflows.

\section{References}

1. Audette, M.A., Ferrie, F.P., Peters, T.M.: An algorithmic overview of surface registration techniques for medical imaging. Med Image Anal 4(3) (2000) 201217

2. Salvi, J., Matabosch, C., Fofi, D., Forest, J.: A review of recent range image registration methods with accuracy evaluation. Image Vis Comput 25(5) (2007) $578-596$

3. van Kaick, O., Zhang, H., Hamarneh, G., Cohen-Or, D.: A survey on shape correspondence. Computer Graphics Forum 30(6) (2011) 1681-1707

4. Heimann, T., Meinzer, H.P.: Statistical shape models for 3D medical image segmentation: A review. Med Image Anal 13(4) (2009) 543-563

5. Sotiras, A., Christos, D., Paragios, N.: Deformable medical image registration: A survey. Research Report RR-7919, INRIA (2012)

6. Schaller, C., Rohkohl, C., Penne, J., Stürmer, M., Hornegger, J.: Inverse C-arm positioning for interventional procedures using real-time body part detection. In Yang, G.Z., et al., eds.: MICCAI, Part I. LNCS 5761, Springer (2009)

7. Grimm, R., Bauer, S., Sukkau, J., Hornegger, J., Greiner, G.: Markerless estimation of patient orientation, posture and pose using range and pressure imaging. Int J Comput Assist Radiol Surg 7(6) (2012) 921-929

8. Bauer, S., Wasza, J., Haase, S., Marosi, N., Hornegger, J.: Multi-modal surface registration for markerless initial patient setup in radiation therapy using Microsoft's Kinect sensor. In: ICCV Workshop on Consumer Depth Cameras for Computer Vision, IEEE (2011) 1175-1181

9. Schöffel, P.J., Harms, W., Sroka-Perez, G., Schlegel, W., Karger, C.P.: Accuracy of a commercial optical 3D surface imaging system for realignment of patients for radiotherapy of the thorax. Phys Med Biol 52(13) (2007) 3949-3963

10. Placht, S., Stancanello, J., Schaller, C., Balda, M., Angelopoulou, E.: Fast timeof-flight camera based surface registration for radiotherapy patient positioning. Med Phys 39(1) (2012) 4-17

11. Wasza, J., Bauer, S., Hornegger, J.: Real-time motion compensated patient positioning and non-rigid deformation estimation using 4-D shape priors. In: MICCAI, Part II. LNCS 7511, Springer (2012) 576-583

12. Lindl, B.L., Müller, R.G., Lang, S., Lablanca, M.D.H., Klöck, S.: Topos: A new topometric patient positioning and tracking system for radiation therapy based on structured white light. Med Phys 40(4) (2013) 042701 
13. Brahme, A., Nyman, P., Skatt, B.: 4D laser camera for accurate patient positioning, collision avoidance, image fusion and adaptive approaches during diagnostic and therapeutic procedures. Med Phys 35(5) (2008) 1670-1681

14. Ettl, S., Fouladi-Movahed, S., Bauer, S., Arold, O., Willomitzer, F., Huber, F., Rampp, S., Stefan, H., Hornegger, J., Häusler, G.: Medical applications enabled by a motion-robust optical 3D sensor. In: DGaO Conference. (2012)

15. Schaller, C., Adelt, A., Penne, J., Hornegger, J.: Time-of-flight sensor for patient positioning. In Samei, E., Hsieh, J., eds.: SPIE Medical Imaging. Volume 7258. (2009) 726110

16. Besl, J., McKay, N.: A method for registration of 3-D shapes. IEEE Trans Pattern Anal Mach Intell 14(2) (1992) 239-256

17. Chen, Y., Medioni, G.: Object modelling by registration of multiple range images. Image Vis Comput 10(3) (1992) 145-155

18. Bauer, S., Berkels, B., Ettl, S., Arold, O., Hornegger, J., Rumpf, M.: Marker-less reconstruction of dense 4-D surface motion fields using active laser triangulation for respiratory motion management. In: MICCAI, Part I. LNCS 7511, Springer (2012) 414-421

19. Keall, P.J., Mageras, G.S., Balter, J.M., Emery, R.S., Forster, K.M., Jiang, S.B., Kapatoes, J.M., Low, D.A., Murphy, M.J., Murray, B.R., Ramsey, C.R., Herk, M.B.V., Vedam, S.S., Wong, J.W., Yorke, E.: The management of respiratory motion in radiation oncology report of AAPM task group 76. Med Phys 33(10) (2006) 3874-3900

20. Verellen, D., Depuydt, T., Gevaert, T., Linthout, N., Tournel, K., Duchateau, M., Reynders, T., Storme, G., Ridder, M.D.: Gating and tracking, 4D in thoracic tumours. Cancer Radiother 14(67) (2010) 446-454

21. Schaller, C., Penne, J., Hornegger, J.: Time-of-Flight Sensor for Respiratory Motion Gating. Med Phys 35(7) (2008) 3090-3093

22. Xia, J., Siochi, R.A.: A real-time respiratory motion monitoring system using kinect: Proof of concept. Med Phys 39(5) (2012) 2682-2685

23. Alnowami, M., Alnwaimi, B., Tahavori, F., Copland, M., Wells, K.: A quantitative assessment of using the kinect for Xbox360 for respiratory surface motion tracking. In: SPIE Medical Imaging. (2012) 83161T-10

24. Yan, H., Yin, F.F., Zhu, G.P., Ajlouni, M., Kim, J.H.: The correlation evaluation of a tumor tracking system using multiple external markers. Med Phys 33(11) (2006) 4073-4084

25. Fayad, H., Pan, T., Clement, J.F., Visvikis, D.: Correlation of respiratory motion between external patient surface and internal anatomical landmarks. Med Phys 38(6) (2011) 3157-3164

26. McClelland, J., Hawkes, D., Schaeffter, T., King, A.: Respiratory motion models: A review. Med Image Anal 17(1) (2013) 19-42

27. Bauer, S., Berkels, B., Hornegger, J., Rumpf, M.: Joint ToF image denoising and registration with a ct surface in radiation therapy. In Bruckstein, A., ter Haar Romeny, B., Bronstein, A., Bronstein, M., eds.: SSVM. LNCS 6667. Springer (2012) 98-109

28. Schaerer, J., Fassi, A., Riboldi, M., Cerveri, P., Baroni, G., Sarrut, D.: Multidimensional respiratory motion tracking from markerless optical surface imaging based on deformable mesh registration. Phys Med Biol 57(2) (2012) 357-373

29. Bauer, S., Wasza, J., Hornegger, J.: Photometric estimation of 3D surface motion fields for respiration management. In Tolxdorff, T., Deserno, T.M., Handels, H., Meinzer, H.P., eds.: Bildverarbeitung für die Medizin. Springer (2012) 105-110 
30. Wasza, J., Bauer, S., Haase, S., Hornegger, J.: Sparse principal axes statistical surface deformation models for respiration analysis and classification. In Tolxdorff, T., Deserno, T.M., Handels, H., Meinzer, H.P., eds.: Bildverarbeitung für die Medizin. Springer (2012) 316-321

31. Gianoli, C., Riboldi, M., Spadea, M.F., Travaini, L.L., Ferrari, M., Mei, R., Orecchia, R., Baroni, G.: A multiple points method for 4D CT image sorting. Med Phys 38(2) (2011) 656-667

32. Bettinardi, V., Bernardi, E.D., Presotto, L., Gilardi, M.: Motion-tracking hardware and advanced applications in PET and PET/CT. PET Clinics 8(1) (2013) $11-28$

33. Alnowami, M.R., Lewis, E., Guy, M., Wells, K.: An observation model for motion correction in nuclear medicine. In: SPIE Medical Imaging. (2010) 76232F-9

34. Bruyant, P., Gennert, M.A., Speckert, G., Beach, R., Morgenstern, J., Kumar, N., Nadella, S., King, M.: A robust visual tracking system for patient motion detection in SPECT: hardware solutions. IEEE Trans Nucl Sci 52(5) 1288-1294

35. McNamara, J.E., Pretorius, P.H., Johnson, K., Mukherjee, J.M., Dey, J., Gennert, M.A., King, M.A.: A flexible multicamera visual-tracking system for detecting and correcting motion-induced artifacts in cardiac SPECT slices. Med Phys 36(5) (2009) 1913-1923

36. Olesen, O.V., Jorgensen, M.R., Paulsen, R.R., Hojgaard, L., Roed, B., Larsen, R.: Structured light 3D tracking system for measuring motions in PET brain imaging. In: SPIE Medical Imaging. (2010) 76250X-11

37. Noonan, P., Howard, J., Tout, D., Armstrong, I., Williams, H., Cootes, T., Hallett, W., Hinz, R.: Accurate markerless respiratory tracking for gated whole body PET using the Microsoft Kinect. In: IEEE NSS-MIC. (2012)

38. Cash, D.M., Sinha, T.K., Chapman, W.C., Terawaki, H., Dawant, B.M., Galloway, R.L., Miga, M.I.: Incorporation of a laser range scanner into image-guided liver surgery: Surface acquisition, registration, and tracking. Med Phys 30(7) (2003) $1671-1682$

39. Cash, D.M., Miga, M.I., Glasgow, S.C., Dawant, B.M., Clements, L.W., Cao, Z., Galloway, R.L., Chapman, W.C.: Concepts and preliminary data toward the realization of image-guided liver surgery. J Gastrointest Surg 11 (2007) 844-859

40. Cash, D.M., Miga, M.I., Sinha, T.K., Galloway, R.L., Chapman, W.C.: Compensating for intraoperative soft-tissue deformations using incomplete surface data and finite elements. IEEE Trans Med Imaging 24(11) (Nov 2005) 1479-1491

41. Seitel, A.: Markerless Navigation For Percutaneus Needle Insertions. PhD thesis, Universität Heidelberg (2012)

42. Mersmann, S., Müller, M., Seitel, A., Arnegger, F., Tetzlaff, R., Dinkel, J., Baumhauer, M., Schmied, B., Meinzer, H.P., Maier-Hein, L.: Time-of-flight camera technology for augmented reality in computer-assisted interventions. In Wong, K.H., Holmes, D.R., eds.: SPIE Medical Imaging. (2011) 79642C

43. Baumhauer, M., Simpfendörfer, T., Stich, B.M., Teber, D., Gutt, C., Rassweiler, J., Meinzer, H.P., Wolf, I.: Soft tissue navigation for laparoscopic partial nephrectomy. Int J Comput Assist Radiol Surg 3 (2008) 307-314

44. dos Santos, T.R.: Muti-Modal Partial Surface Matching For Intraoperative Registration. PhD thesis, Universität Heidelberg (2012)

45. Wang, X.L., Stolka, P.J., Boctor, E., Hager, G., Choti, M.: The Kinect as an interventional tracking system. In: SPIE Medical Imaging. (2012) 83160U-6

46. Nicolau, S., Brenot, J., Goffin, L., Graebling, P., Soler, L., Marescaux, J.: A structured light system to guide percutaneous punctures in interventional radiology. In: SPIE Medical Imaging. (2008) 700016 
47. Mirota, D.J., Ishii, M., Hager, G.D.: Vision-based navigation in image-guided interventions. Annu Rev Biomed Eng 13(13) (2011) 297-319

48. Stoyanov, D., Mylonas, G., Deligianni, F., Darzi, A., Yang, G.: Soft-tissue motion tracking and structure estimation for robotic assisted MIS procedures. In: MICCAI, Part II. LNCS 3750, Springer (2005) 139-146

49. Collins, T., Bartoli, A.: Live monocular 3D laparoscopy using shading and specularity information. In: IPCAI. LNAI 7330, Springer (2012) 11-21

50. Malti, A., Bartoli, A., Collins, T.: Template-based conformal shape-from-motion for laparoscopy. In: ICPAI. LNAI 7330, Springer (2012) 1-10

51. Mountney, P., Stoyanov, D., Yang, G.Z.: Three-dimensional tissue deformation recovery and tracking. IEEE Signal Proc Mag 27 (2010) 14-24

52. Clancy, N.T., Stoyanov, D., Yang, G.Z., Elson, D.S.: An endoscopic structured lighting probe using spectral encoding. In: SPIE Novel Biophotonic Techniques and Applications. Volume 8090. (2011)

53. Schmalz, C., Forster, F., Schick, A., Angelopoulou, E.: An endoscopic 3D scanner based on structured light. Med Image Anal 16(5) (2012) 1063-1072

54. Maier-Hein, L., Mountney, P., Bartoli, A., Elhawary, H., Elson, D., Groch, A., Kolb, A., Rodrigues, M., Sorger, J., Speidel, S., Stoyanov, D.: Optical techniques for 3D surface reconstruction in computer-assisted laparoscopic surgery. Med Image Anal (2013) (in press)

55. Penne, J., Höller, K., Stürmer, M., Schrauder, T., Schneider, A., Engelbrecht, R., Feußner, H., Schmauss, B., Hornegger, J.: Time-of-flight 3-D endoscopy. In Yang, G.Z., et al., eds.: MICCAI, Part I. LNCS 5761 (2009) 467-474

56. Groch, A., Seitel, A., Hempel, S., Speidel, S., Engelbrecht, R., Penne, J., Höller, K., Röhl, S., Yung, K., Bodenstedt, S., Pflaum, F., dos Santos, T., Mersmann, S., Meinzer, H.P., Hornegger, J., Maier-Hein, L.: 3D surface reconstruction for laparoscopic computer-assisted interventions: Comparison of state-of-the-art methods. In: SPIE Medical Imaging. (2011) 796415

57. Haase, S., Wasza, J., Kilgus, T., Hornegger, J.: Laparoscopic instrument localization using a 3-D Time-of-Flight/RGB endoscope. In: Workshop on the Applications of Computer Vision, IEEE (2013) 449-454

58. Groch, A., Haase, S., Wagner, M., Kilgus, T., Kenngott, H., Schlemmer, H.P., Hornegger, J., Meinzer, H.P., Maier-Hein, L.: A probabilistic approach to fusion of Time-of-Flight and multiple view based 3D surface reconstruction for laparoscopic interventions. In: Int J Comput Assist Radiol Surg. Volume 7. (2012) S397-S398

59. Kolb, C., Groch, A., Seitel, A., Kilgus, T., Haase, S., Bendl, R., Meinzer, H.P., Hornegger, J., Maier-Hein, L.: Simultaneous localization and soft-tissue shape recovery with a time of flight endoscope for computer-assisted surgery. In: Int J Comput Assist Radiol Surg. (2013) (in press)

60. Ladikos, A., Benhimane, S., Navab, N.: Real-time 3D reconstruction for collision avoidance in interventional environments. In: MICCAI, Part II. LNCS 5242, Springer (2008) 526-534

61. Navab, N., Holzer, S.: Real-time 3D reconstruction: applications to collision detection and surgical workflow monitoring. In: IROS Workshop on Methods for Safer Surgical Robotics Procedures. (2011)

62. SAFROS project: http://www.safros.eu/

63. ACTIVE project: http://www.active-fp7.eu/

64. Mönnich, H., Nicolai, P., Raczkowsky, J., Wörn, H.: A semi-autonomous robotic teleoperation surgery setup with multi 3D camera supervision. In: Int J Comput Assist Radiol Surg. (2011) 132-133 
65. Nicolai, P., Raczkowsky, J.: Operation room supervision for safe robotic surgery with a multi 3D-camera setup. In: IROS Workshop on Methods for Safer Surgical Robotics Procedures. (2011)

66. Katic, D., Wekerle, A.L., Gärtner, F., Kenngott, H., Müller-Stich, B.P., Dillmann, R., Speidel, S.: Ontology-based prediction of surgical events in laparoscopic surgery. In: SPIE Medical Imaging. (2013) 86711A-7

67. Padoy, N., Mateus, D., Weinland, D., Berger, M.O., Navab, N.: Workflow monitoring based on 3D motion features. In: ICCV Workshop on Video-oriented Object and Event Classification, IEEE (2009) 585-592

68. Lea, C.S., Fackler, J.C., Hager, G.D., , Taylor, R.H.: Towards automated activity recognition in an intensive care unit. In: MICCAI Workshop on Modeling and Monitoring of Computer Assisted Interventions. (2012) 19-28

69. Ladikos, A., Cagniart, C., Ghotbi, R., Reiser, M., Navab, N.: Estimating radiation exposure in interventional environments. In: MICCAI, Part III. LNCS 6363, Springer (2010) 237-244

70. Gratzel, C., Fong, T., Grange, S., Baur, C.: A non-contact mouse for surgeoncomputer interaction. Technology and Health Care - European Society for Engineering and Medicine 12(3) (2004) 245-258

71. Soutschek, S., Penne, J., Hornegger, J., Kornhuber, J.: 3-D gesture-based scene navigation in medical imaging applications using Time-Of-Flight cameras. In: CVPR Workshop on Time of Flight Camera based Computer Vision, IEEE (2008) $1-6$

72. Ruppert, G., Reis, L., Amorim, P., de Moraes, T., da Silva, J.: Touchless gesture user interface for interactive image visualization in urological surgery. World $\mathrm{J}$ Urol 30 (2012) 1-5

73. Gallo, L., Placitelli, A.P., Ciampi, M.: Controller-free exploration of medical image data: Experiencing the Kinect. In: International Symposium on ComputerBased Medical Systems, IEEE (2011) 1-6

74. Jacob, M., Cange, C., Packer, R., Wachs, J.: Intention, context and gesture recognition for sterile MRI navigation in the operating room. Progress in Pattern Recognition, Image Analysis, Computer Vision, and Applications 7441 (2012) $220-227$

75. Kirmizibayrak, C., Radeva, N., Wakid, M., Philbeck, J., Sibert, J., Hahn, J.: Evaluation of gesture based interfaces for medical volume visualization tasks. In: International Conference on Virtual Reality Continuum and Its Applications in Industry, ACM (2011) 69-74

76. Ebert, L., Hatch, G., Ampanozi, G., Thali, M., Ross, S.: You can't touch this: Touch-free navigation through radiological images. Surg Innov 19(3) (2012) 301307

77. Bigdelou, A., Stauder, R., Benz, T., Okur, A., Blum, T., Ghotbi, R., Navab, N.: HCI design in the OR: A gesturing case-study. In: MICCAI Workshop on Modeling and Monitoring of Computer Assisted Interventions, Springer (2012) $10-18$

78. Karl Storz GmbH, Tuttlingen, Germany: http://www.mi-report.com

79. Bigdelou, A., Benz, T., Schwarz, L., Navab, N.: Simultaneous categorical and spatio-temporal 3D gestures using Kinect. In: Symposium on 3D User Interfaces, IEEE (2012) 53-60

80. Dressler, C., Neumuth, T., Fischer, M., Abri, O., Strauss, G.: Intraoperative Bedienung einer elektronischen Patientenakte durch den Operateur. HNO 59(9) (2011) 900-907 
81. Navab, N., Traub, J., Sielhorst, T., Feuerstein, M., Bichlmeier, C.: Action- and workflow-driven augmented reality for computer-aided medical procedures. IEEE Comput Graph Appl 27(5) (2007) 10-14

82. Sugimoto, M., Yasuda, H., Koda, K., Suzuki, M., Yamazaki, M., Tezuka, T., Kosugi, C., Higuchi, R., Watayo, Y., Yagawa, Y., Uemura, S., Tsuchiya, H., Azuma, T.: Image overlay navigation by markerless surface registration in gastrointestinal, hepatobiliary and pancreatic surgery. J Hepatobiliary Pancreat Sci 17(5) (2010) 629-636

83. Maier-Hein, L., Franz, A.M., Fangerau, M., Schmidt, M., Seitel, A., Mersmann, S., Kilgus, T., Groch, A., Yung, K., dos Santos, T.R., Meinzer, H.P.: Towards mobile augmented reality for on-patient visualization of medical images. In: Bildverarbeitung für die Medizin, Springer (2011) 389-393

84. Blum, T., Kleeberger, V., Bichlmeier, C., Navab, N.: mirracle: An augmented reality magic mirror system for anatomy education. In: Virtual Reality, IEEE (2012) 115-116

85. Maier-Hein, L., Schmidt, M., Franz, A., dos Santos, T., Seitel, A., Jähne, B., Fitzpatrick, J., Meinzer, H.: Accounting for anisotropic noise in fine registration of time-of-flight range data with high-resolution surface data. In: MICCAI, Part I. LNCS 6361, Springer (2010) 251-258

86. Maier-Hein, L., Franz, A., dos Santos, T., Schmidt, M., Fangerau, M., Meinzer, H.P., Fitzpatrick, J.M.: Convergent iterative closest-point algorithm to accomodate anisotropic and inhomogenous localization error. IEEE Trans Pattern Anal Mach Intell 34(8) (2012) 1520-1532

87. Kilgus, T., Franz, A.M., Seitel, A., März, K., Bartha, L., Fangerau, M., Mersmann, S., Groch, A., Meinzer, H.P., Maier-Hein, L.: Registration of partially overlapping surfaces for range image based augmented reality on mobile devices. In: SPIE Medical Imaging. (2012) 83160T

88. Gabel, M., Gilad-Bachrach, R., Renshaw, E., Schuster, A.: Full body gait analysis with Kinect. In: International Conference of Engineering in Medicine and Biology Society, IEEE (2012) 1964-1967

89. Parra-Dominguez, G., Taati, B., Mihailidis, A.: 3D human motion analysis to detect abnormal events on stairs. In: International Conference on 3D Imaging, Modeling, Processing, Visualization and Transmission. (2012) 97-103

90. Garcia, J.A., Navarro, K.F., Schoene, D., Smith, S.T., Pisan, Y.: Exergames for the elderly: towards an embedded Kinect-based clinical test of falls risk. Studies in Health Technology and Informatics. In: Health Informatics: Building a Healthcare Future Through Trusted Information. IOS (2012) 51-57

91. Parajuli, M., Tran, D., Ma, W., Sharma, D.: Senior health monitoring using Kinect. In: International Conference on Communications and Electronics. (2012) 309-312

92. Stone, E., Skubic, M.: Evaluation of an inexpensive depth camera for in-home gait assessment. J Ambient Intell Smart Environ 3(4) (2011) 349-361

93. Stone, E., Skubic, M.: Passive in-home measurement of stride-to-stride gait variability comparing vision and Kinect sensing. In: International Conference of Engineering in Medicine and Biology Society. (2011) 6491-6494

94. Gross, H., Schroeter, C., Mueller, S., Volkhardt, M., Einhorn, E., Bley, A., Martin, C., Langner, T., Merten, M.: Progress in developing a socially assistive mobile home robot companion for the elderly with mild cognitive impairment. In: IROS, IEEE/RSJ (2011) 2430-2437 
95. Lowet, D., Isken, M., Lee, W., van Heesch, F., Eertink, E.: Robotic telepresence for 24/07 remote assistance to elderly at home, workshop on social robotic telepresence. In: International Symposium on Robot and Human Interactive Communication, IEEE (2012)

96. Woo, J., Wada, K., Kubota, N.: Robot partner system for elderly people care by using sensor network. In: International Conference on Biomedical Robotics and Biomechatronics, IEEE, RAS, EMBS (2012) 1329-1334

97. Shotton, J., Girshick, R., Fitzgibbon, A., Sharp, T., Cook, M., Finocchio, M., Moore, R., Kohli, P., Criminisi, A., Kipman, A., Blake, A.: Efficient human pose estimation from single depth images. IEEE Trans Pattern Anal Mach Intell 99(PrePrints) (2012)

98. Ganapathi, V., Plagemann, C., Koller, D., Thrun, S.: Real time motion capture using a single Time-of-Flight camera. In: CVPR, IEEE (2010) 755-762

99. Schwarz, L., Mkhitaryan, A., Mateus, D., Navab, N.: Estimating human 3D pose from Time-of-Flight images based on geodesic distances and optical flow. In: International Conference on Automatic Face Gesture Recognition and Workshops, IEEE (2011) 700-706

100. Coronato, A., Gallo, L.: Towards abnormal behavior detection of cognitive impaired people. In: International Conference on Pervasive Computing and Communications Workshops, IEEE (2012) 859-864

101. Sivalingam, R., Cherian, A., Fasching, J., Walczak, N., Bird, N.D., Morellas, V., Murphy, B., Cullen, K., Lim, K., Sapiro, G., Papanikolopoulos, N.: A multisensor visual tracking system for behavior monitoring of at-risk children. In: ICRA. (2012) 1345-1350

102. Walczak, N., Fasching, J., Toczyski, W.D., Sivalingam, R., Bird, N.D., Cullen, K., Morellas, V., Murphy, B., Sapiro, G., Papanikolopoulos, N.: A nonintrusive system for behavioral analysis of children using multiple RGB+depth sensors. In: Workshop on the Applications of Computer Vision. (2012) 217-222

103. Falie, D., Ichim, M., David, L.: Respiratory motion visualization and the sleep apnea diagnosis with the time of flight ( ToF) camera. In: International Conference on Visualization, Imaging and Simulation, WSEAS (2008) 179-184

104. Yu, M.C., Wu, H., Liou, J.L., Lee, M.S., Hung, Y.P.: Breath and position monitoring during sleeping with a depth camera. In: HEALTHINF. (2012) 12-22

105. Smith, S.T., Schoene, D.: The use of exercise-based videogames for training and rehabilitation of physical function in older adults: current practice and guidelines for future research. Aging Health 8(3) (2012) 243-252

106. Virtualware Group, Basauri, Spain: http://virtualrehab.info/en/

107. Jintronix, Inc., Montreal, QC, Canada: http://www.jintronix.com/.

108. Chang, Y.J., Chen, S.F., Huang, J.D.: A Kinect-based system for physical rehabilitation: A pilot study for young adults with motor disabilities. Research in Developmental Disabilities 32(6) (2011) 2566-2570

109. da Gama, A., Chaves, T., Figueiredo, L., Teichrieb, V.: Improving motor rehabilitation process through a natural interaction based system using Kinect sensor. In: Symposium on 3D User Interfaces, IEEE (2012) 145-146

110. Huang, J.D.: Kinerehab: a Kinect-based system for physical rehabilitation: a pilot study for young adults with motor disabilities. In: International ACM SIGACCESS Conference on Computers and Accessibility. ASSETS (2011) 319-320

111. Soutschek, S., Maier, A., Bauer, S., Kugler, P., Bebenek, M., Steckmann, S., von Stengel, S., Kemmler, W., Hornegger, J., Kornhuber, J.: Measurement of angles in Time-of-Flight data for the automatic supervision of training exercises. In: 
Conference on Pervasive Computing Technologies for Healthcare, IEEE (2010) $1-4$

112. Schoenauer, C., Pintaric, T., Kaufmann, H., Jansen Kosterink, S., VollenbroekHutten, M.: Chronic pain rehabilitation with a serious game using multimodal input. In: International Conference on Virtual Rehabilitation. (2011) 1-8

113. Chang, C.Y., Lange, B., Zhang, M., Koenig, S., Requejo, P., Somboon, N., Sawchuk, A.A., Rizzo, A.A.: Towards pervasive physical rehabilitation using Microsoft Kinect. In: International Conference on Pervasive Computing Technologies for Healthcare. (2012) 159-162

114. Lange, B., Chang, C.Y., Suma, E., Newman, B., Rizzo, A., Bolas, M.: Development and evaluation of low cost game-based balance rehabilitation tool using the Microsoft Kinect sensor. In: International Conference of Engineering in Medicine and Biology Society, IEEE (2011) 1831-1834

115. Hersh, M., Johnson, M., Keating, D.: Assistive Technology for Visually Impaired and Blind People. Springer (2007)

116. Gallo, S., Chapuis, D., Santos-Carreras, L., Kim, Y., Retornaz, P., Bleuler, H., Gassert, R.: Augmented white cane with multimodal haptic feedback. In: International Conference on Biomedical Robotics and Biomechatronics, IEEE, RAS, EMBS (2010) 149-155

117. Gassert, R., Kim, Y., Oggier, T., Riesch, M., Deschler, M., Prott, C., Schneller, S., Hayward, V.: White cane with integrated electronic travel aid using 3D TOF sensor (2012) Patent WO 2012/040703.

118. Katz, B., Kammoun, S., Parseihian, G., Gutierrez, O., Brilhault, A., Auvray, M., Truillet, P., Denis, M., Thorpe, S., Jouffrais, C.: Navig: augmented reality guidance system for the visually impaired. Virtual Reality 16 (2012) 253-269

119. Ong, S.K., Zhang, J., Nee, A.Y.C.: Assistive obstacle detection and navigation devices for vision-impaired users. Disability and Rehabilitation: Assistive Technology (2013) (Epub ahead of print)

120. IS2you, Santa Maria, Portugal: http://www.is2you.eu/eng/products.html.

121. Buttgen, B.: Extending Time-of-Flight optical 3D-imaging to extreme operating conditions. PhD thesis, Universite de Neuchatel (2007)

122. Maimone, A., Fuchs, H.: Reducing interference between multiple structured light depth sensors using motion. In: Virtual Reality, IEEE (2012) 51-54

123. Roggan, A., Friebel, M., Dörschel, K., Hahn, A., Müller, G.: Optical properties of circulating human blood in the wavelength range 400-2500 nm. J Biomed Opt 4(1) (1999) 36-46

124. Fuchs, S.: Multipath interference compensation in time-of-flight camera images. In: ICPR. (2010) 3583-3586

125. Dorrington, A.A., Godbaz, J.P., Cree, M.J., Payne, A.D., Streeter, L.V.: Separating true range measurements from multi-path and scattering interference in commercial range cameras. In: SPIE Electronic Imaging. (2011) 786404-10

126. Wu, D., O'Toole, M., Velten, A., Agrawal, A., Raskar, R.: Decomposing global light transport using time of flight imaging. In: CVPR, IEEE (2012) 366-373

127. Bert, C., Metheany, K.G., Doppke, K., Chen, G.T.Y.: A phantom evaluation of a stereo-vision surface imaging system for radiotherapy patient setup. Med Phys 32(9) (Sep 2005) 2753-2762

128. Lange, R.: 3D Time-of-Flight Distance Measurement with Custom Solid-State Image Sensors in CMOS/CCD-Technology. PhD thesis, University of Siegen (2000)

129. Seitel, A., Yung, K., Mersmann, S., Kilgus, T., Groch, A., Santos, T., Franz, A., Nolden, M., Meinzer, H.P., Maier-Hein, L.: MITK-ToF - range data within MITK. Int J Comput Assist Radiol Surg 7 (2012) 87-96 
130. Wolf, I., Vetter, M., Wegner, I., Böttger, T., Nolden, M., Schöbinger, M., Hastenteufel, M., Kunert, T., Meinzer, H.P.: The medical imaging interaction toolkit. Med Image Anal 9(6) (2005) 594-604

131. Wasza, J., Bauer, S., Haase, S., Schmid, M., Reichert, S., Hornegger, J.: RITK: The range imaging toolkit - a framework for 3-D range image stream processing. In Eisert, P., Hornegger, J., Polthier, K., eds.: International Workshop on Vision, Modeling and Visualization. (2011) 57-64

132. Ibanez, L., Schroeder, W., Ng, L., Cates, J.: The ITK Software Guide. Kitware, Inc. 2nd edn. (2005)

133. Rusu, R.B., Cousins, S.: 3D is here: Point cloud library (PCL). In: ICRA. (2011) $1-4$ 